\title{
Editing gene engineering to enhance function
}

\author{
Emma C. Morris | University College London
}

In this issue of Blood, Legut et al have tackled a major scientific challenge relating to T-cell engineering, with an unexpected outcome. They have demonstrated that CRISPR-mediated knockout of the native T-cell receptor (TCR) $\beta$ chain dramatically improves the expression of inserted therapeutic $\alpha \beta$ and $\gamma \delta$ TCRs in comparison with the same TCRs inserted without endogenous TCR- $\beta$ chain knockout. Although insertion of TCRs or chimeric antigen receptors (CARs) into the TCR locus has been reported previously, the significant improvement in functional avidity of TCR modified T cells shown here is novel.1

Gene and cell therapy has arrived in the mainstream. With the recent US Food and Drug Administration (FDA) approval of 2 genetically engineered T-cellproducts, tisagenlecleucel (Kymriah) 2 and axicabtagene ciloleucel (Yescarta),3 cellular immunotherapy is finally coming of age. Geneengineered T cells are one of the "newest kids on the block," and they are generating much excitement. However, CD19-specific CART cells are the tip of an enormous iceberg. Following behind in early-phase clinical trials are second- and third-generation products and a growing number of TCR gene-modified T cells, all designed to improve both efficacy and safety profiles. Although CAR recognition is limited to cell surface structures, TCR can recognize intracellular proteins. This not only expands the range of tumor-associated self-antigens that are amenable to T-cell therapy, but it also allows TCR targeting of the cancer mutagenome, ${ }^{4}$ which may be more important when targeting solid tumors.

Autologous T cells isolated from the peripheral T-cell repertoire are the starting material for most engineered T-cell products. These T cells express their own endogenous TCRs (either $\alpha \beta T C R$ or $Y \delta T C R$ heterodimers) with a predetermined antigen specificity. Upon introduction of an additional therapeutic TCR, there is competition with the endogenous TCR for self-surface expression. During TCR assembly within the endoplasmic reticulum, both endogenous and therapeutic TCR chains are available for assembly into the $\alpha \beta T C R-C D 3$ complex prior to transport to the cell surface. Cell surface expression of hybrid TCRs due to mispairing of endogenous $\alpha$ and therapeutic/introduced $\beta$ TCR chains or vice versa (so-called mixed TCR dimers) may therefore occur.

As Legut et al point out in the introduction to their article, a TCR-transduced T cell therefore has the potential to express four distinct TCRs, only one of which is desired. Mixed TCR dimers can exhibit unpredictable, and potentially dangerous, target specificities. Although clinical trials have not yet demonstrated toxicity due to TCR mispairing, it is clear from murine model experiments that it can cause severe graftversus-host disease-like pathology. ${ }^{5,6}$ A number of strategies are being explored to optimize expression of the therapeutic TCR and reduce mispairing, including the provision of additional $C D 3,{ }^{7}$ the modification of TCR sequences to enhance expression, and knockdown of the endogenous TCR.

The ability to knock down or suppress the endogenous TCR repertoire during the process of Tcell engineering to redirect antigen specificity is important to (a) increase expression of introduced TCR, (b) reduce risk of TCR mispairing and thus generation of T cells with unknown and

$$
\text { potentially }
$$

(c) permit the

$$
\text { autoreactive }
$$

allogeneic T-cell

safe use

donors

$\begin{array}{cc}\text { specificities, } & \text { and } \\ \text { third-party } & \text { or } \\ \text { (relevant } & \text { for }\end{array}$

both CAR and TCR engineering). ${ }^{8}$ 
Meet the other "new kids on the block": gene editing tools such as CRISPR-Cas9, TALENs, and MegaTAL nucleases, which are all being evaluated for their ability to reliably and efficiently edit primary human T cells. In this issue of Blood, Legut et al describe a strategy to simultaneously knock down the endogenous TCR- $\beta$ chain in recipient T cells using CRISPR/Cas 9 technology while transducing a cancer-reactive TCR of choice. ${ }^{4}$

There have been several previously reported attempts to combine endogenous TCR knockout, using zinc finger nucleases, ${ }^{9}$ transcription activator-like effector nucleases, ${ }^{8}$ or CRISPR/Cas9, ${ }^{10}$ with redirecting the T cells to cancer, in most cases via CARs. Others have used targeted insertion of a CAR into the TCR a locus and demonstrated improved function of the engineered T cell.

However, this article goes further in demonstrating that the cell surface expression of introduced antigen-specific TCRs ( $\alpha \beta T C R$ or $\gamma \delta T C R$ ) can be significantly enhanced by the simultaneous disruption of the endogenous TCR-b chain, in comparison with the expression levels achieved for the same TCRs inserted without endogenous TCR- $\beta$ chain knockout. This "TCR replacement" strategy translated to an enhancement of in vitro function assessed by CD107 expression, interferon $\mathrm{g}$, and tumor necrosis factor a secretion.

The subsequent focus of this article was on therapeutic $\gamma \delta T C R s$. The $\gamma \delta T C R$ as a cancer-reactive reagent has a number of potential advantages over the $\alpha \beta T C R$, including no requirement for HLA restriction and $\gamma \delta T C R$ targets that are expressed by a broad range of cancers. The authors successfully redirected primary $T$ cells with a pancancer-reactive $\gamma \delta T C R(\gamma \delta 20)$ in combination with endogenous TCR- $\beta$ knockout and again demonstrated that removal of the endogenous TCR- $\beta$ chain led to a striking increase of surface expression of the $\gamma \delta 20$ TCR and also increased peptide sensitivity by several orders of magnitude.

Widespread clinical application of gene-editing technology is just around the corner, as is demonstrated by the recent success of the off-the-shelf allogeneic CAR19 T cells in inducing remission of B-ALL in an infant. ${ }^{8}$ Just as we become comfortable with the idea of prescribing our patients gene-engineered cell products, we are already looking to the future, where combinations of gene engineering and gene editing are predicted to optimize the function and safety of a cellular product.

\section{REFERENCES}

1. Legut M, Dolton G, Mian AA, Ottmann OG, Sewell AK. CRISPR-mediated TCR replace-ment generates superior anticancer trans-genic T cells. Blood. 2018;131(3):311-322.

2. Novartis. Novartis receives first ever FDA approval for a CAR-T cell therapy, KymriahTTM (tisagenlecleucel, CTL019), for children and young adults with B-cell ALL that is refractory or has relapsed at least twice [press release]. https://novartis.gcs-web.com/novartisreceivesfda-approval-for-KymriahTM. Accessed 30 August 2017.

3. Gilead. Kite's YescartaTTM (Axicabtagene Ciloleucel) becomes first CAR T therapy approved by the FDA for the treatment of adult patients with relapsed or refractory large B-cell lymphoma after two or more lines of systemic therapy [press release]. http://www.businesswire. 
com/news/home/20171018006639/en/. Accessed 18 October 2017.

4. Morris EC, Stauss HJ. Optimizing T-cell receptor gene therapy for hematologic malignancies. Blood. 2016;127(26): 3305-3311.

5. van Loenen MM, de Boer R, Amir AL, et al. Mixed T cell receptor dimers harbor potentially harmful neoreactivity. Proc Natl Acad Sci USA. 2010;107(24):10972-10977.

6. Bendle GM, Linnemann C, Hooijkaas Al, et al. Lethal graft-versus-host disease in mouse models of T cell receptor gene therapy. Nat Med. 2010;16(5):565-570.

7. Ahmadi M, King JW, Xue SA, et al. CD3 limits the efficacy of TCR gene therapy in vivo. Blood. 2011;118(13): 3528-3537.

8. Qasim W, Zhan H, Samarasinghe S, et al. Molecular remission of infant B-ALL after infusion of universal TALEN gene-edited CAR T cells. Sci. Transl. Med. 2017;9(374): eaaj2013.

9. Provasi E, Genovese P, Lombardo A, et al. Editing T cell specificity towards leukemia by zinc finger nucleases and lentiviral gene transfer. Nat Med. 2012;18(5):807-815.

10. Eyquem J, Mansilla-Soto J, Giavridis T, et al. Targeting a CAR to the TRAC locus with CRISPR/Cas9 enhances tumour rejection. Nature. 2017;543(7643):113-117. 